\title{
Optical Interface States Protected by Synthetic Weyl Points
}

\author{
Qiang Wang, ${ }^{1}$ Meng Xiao, ${ }^{2,3}$ Hui Liu, ${ }^{1, *}$ Shining Zhu, ${ }^{1}$ and C. T. Chan ${ }^{2, \dagger}$ \\ ${ }^{1}$ National Laboratory of Solid State Microstructures, School of Physics, \\ Collaborative Innovation Center of Advanced Microstructures, Nanjing University, \\ Nanjing 210093, China \\ ${ }^{2}$ Department of Physics and the Institute for Advanced Study, \\ The Hong Kong University of Science and Technology, Clear Water Bay, Kowloon, Hong Kong \\ ${ }^{3}$ Department of Electrical Engineering, and Ginzton Laboratory, Stanford University, \\ Stanford, California 94305, USA \\ (Received 30 March 2017; revised manuscript received 9 June 2017; published 16 August 2017)
}

\begin{abstract}
Weyl fermions have not been found in nature as elementary particles, but they emerge as nodal points in the band structure of electronic and classical wave crystals. Novel phenomena such as Fermi arcs and chiral anomaly have fueled the interest in these topological points which are frequently perceived as monopoles in momentum space. Here, we report the experimental observation of generalized optical Weyl points inside the parameter space of a photonic crystal with a specially designed four-layer unit cell. The reflection at the surface of a truncated photonic crystal exhibits phase vortexes due to the synthetic Weyl points, which in turn guarantees the existence of interface states between photonic crystals and any reflecting substrates. The reflection phase vortexes have been confirmed for the first time in our experiments, which serve as an experimental signature of the generalized Weyl points. The existence of these interface states is protected by the topological properties of the Weyl points, and the trajectories of these states in the parameter space resembles those of Weyl semimetal "Fermi arc surface states" in momentum space. Tracing the origin of interface states to the topological character of the parameter space paves the way for a rational design of strongly localized states with enhanced local field.
\end{abstract}

DOI: 10.1103/PhysRevX.7.031032

Subject Areas: Metamaterials, Photonics

\section{INTRODUCTION}

Great effort has been devoted to investigate various intriguing phenomena associated with Weyl points [1-6], such as the Fermi arc surface states [7,8] and the chiral anomaly [2] associated with electronic systems. Besides electronic systems, Weyl points have also been found in photonic [9-14], acoustic [15,16], and plasmonic [17] systems. Up to now, Weyl points have mostly been identified as momentum space magnetic monopoles, i.e., sources or sinks of Berry curvature defined in momentum space. As such, Weyl points are usually perceived as topological nodal points in the 3D momentum space defined by Bloch momentum coordinates $k_{x}, k_{y}$, and $k_{z}$. On the other hand, a few recent works considered the topological singular points in the synthetic dimensions [18-20] instead of the

\footnotetext{
*Corresponding author. liuhui@nju.edu.cn

Corresponding author. phchan@ust.hk

Published by the American Physical Society under the terms of the Creative Commons Attribution 4.0 International license. Further distribution of this work must maintain attribution to the author(s) and the published article's title, journal citation, and DOI.
}

momentum space. The interest in considering synthetic dimensions is fueled by the ability of realizing physics in higher dimensions [18,21-24] and the possibility of simplifying experimental designs [25]. Moreover, the possible control over the synthetic dimensions enables the experimental verification of the nontrivial topology of any closed surface enclosing the topological singular points $[18,19]$. Here, we experimentally realize generalized Weyl points in the optical frequency regime with one-dimensional photonic crystals (PCs) utilizing the concept of synthetic dimensions. Different from previous works [18-20] that replaced all three dimensions with synthetic dimensions, here we replace two wave vector components with two independent geometric parameters (which form a parameter space) in the Weyl Hamiltonian and keep one dimension as the wave vector. By doing this, we retain the novel bulkedge correspondence relation between the edge states and the Weyl points. This is not possible for other topological singular points in purely synthetic dimensions [18-20]. Meanwhile, our hybridized Weyl points also preserve the advantage of using synthetic dimensions, which facilitates the experimental investigation of Weyl physics in the optical region, whose structures are otherwise complex $[11,12,15,26,27]$ and, hence, difficult to fabricate at such frequencies. 
The Weyl Hamiltonian can be written as $H=$ $\sum_{i, j} k_{i} v_{i, j} \sigma_{j}$ [28], where $v_{i, j}, k_{i}$, and $\sigma_{i}$, with $i=x, y, z$, represent the group velocities, wave vectors, and the Pauli matrices, respectively. Unlike 2D Dirac points, Weyl points are stable against perturbations when the wave vectors remain as good quantum numbers and the Weyl points do not interact with other bands [9]. Each Weyl point has its associated topological charge given by the Chern number of a closed surface enclosing it [5]. Though Weyl points are usually defined in the 3D momentum space, very recently Weyl physics has been theoretically discussed in synthetic dimensions [25]. Instead of using all three components of the wave vector, here we replace two wave vector components with two independent geometric parameters, and experimentally investigate the generalized Weyl points with simple 1D PCs. Such generalizations preserve the standard Weyl point characteristics, such as the associated topological charges $[11,13]$ and robustness against variations in the parameters [9].

Moreover, optical interface states can be found between the PCs possessing synthetic Weyl points and any reflection substrates, whose existence is stable and protected by the topological properties of the synthetic Weyl points. We show that the reflection phase of a truncated PC exhibits vortex structures [29] in the parameter space around a synthetic Weyl point. These vortexes carry the same topological charges as the corresponding Weyl points. The reflection phase along any loop in the parameter space enclosing the center of the vortex (also the position of the Weyl point) varies continuously from $-\pi$ to $\pi$. This property guarantees the existence of interface states at the boundary separating the PC and a gapped material such as a reflecting substrate $[30,31]$ independent of the properties of the substrate. The above physical interpretation serves as the bulk-edge correspondence [30,32] for the synthetic Weyl points in our system. These interface states can be regarded as analogues of edge states in Weyl semimetals $[3,6,7]$, and they can be useful in nonlinear optics [33-35], quantum optics [36,37], thermal radiation [38], etc. [39-42]. The winding of the reflection phase has also been discussed in the context of adiabatic charge pumping and Floquet Weyl phases in a three-dimensional network [43-45]. Furthermore, we also introduce a third geometric parameter, which extends the three-dimensional space to four-dimensions. By tuning the third geometric parameter, we also observe the topological transition from Weyl semimetals to nodal line semimetals $[9,46]$.

\section{RESULTS}

\section{A. Synthetic Weyl points in parameter space}

To illustrate the idea of synthetic Weyl points in a generalized parameter space, we consider a 1D PC consisting of four layers per unit cell, as shown in the inset in Fig. 1(a). In our experiments, the first and third layers (blue) are made of $\mathrm{H}_{\mathrm{f}} \mathrm{O}_{2}$ with refractive index $n_{a}=2.00$, and the second and fourth layers (red) are made of $\mathrm{S}_{\mathrm{i}} \mathrm{O}_{2}$ with refractive index $n_{b}=1.45$. The thickness of each layer is given by

$$
\begin{aligned}
& d_{a 1}=(1+p) d_{a}, \\
& d_{b 1}=(1+q) d_{b}, \\
& d_{a 2}=(1-p) d_{a}, \\
& d_{b 2}=(1-q) d_{b} .
\end{aligned}
$$

Since the thickness of each layer cannot be a negative value, $p$ and $q$ both fall in $[-1,1]$, which makes the $p-q$ space a closed parameter space. The total optical length $L$ inside the unit cell is a constant $2\left(n_{a} d_{a}+n_{b} d_{b}\right)$ for the whole $p-q$ space. As illustrated in Fig. 1(a), the structural parameters $p$ and $q$, together with one Bloch wave vector $k$, form a 3D parameter space in which Weyl physics can be studied.

We start with the PCs with only two layers inside each unit cell, a layer of $\mathrm{H}_{\mathrm{f}} \mathrm{O}_{2}$ with thickness $d_{a}$ and a layer of $\mathrm{S}_{\mathrm{i}} \mathrm{O}_{2}$ with thickness $d_{b}$. The band dispersion is plotted in Fig. 1(b) in red. A four-layer PC with parameters $p=0$ and $q=0$ simply doubles the length of each unit cell and folds the Brillouin zone. The dispersion of this four-layer PC is shown in Fig. 1(b) in blue. Such artificial band folding gives a linear crossing along the wave vector direction. Away from the point where $p=0$ and $q=0$, the degeneracy introduced by the band folding is lifted and a band gap emerges. Figure 1(c) shows the band dispersions in the $p-q$ space with $k=0.5 k_{0}$, where $k_{0}=\pi /\left(d_{a}+d_{b}\right)$. Two bands form a conical intersection indicating that band dispersion is linear in all directions. To characterize this degenerate point, we derive an effective Hamiltonian for the parameters around it (see Appendix A):

$$
H=p v_{p z} \sigma_{z}+q v_{q y} \sigma_{y}+\xi_{k} v_{k x} \sigma_{x},
$$

where $\xi_{k}=\left(k-0.5 k_{0}\right) / k_{0}, v_{p z}=0.1073, v_{q y}=-0.0946$, and $v_{k x}=-1.985$ for our system. This Hamiltonian possesses a standard Weyl Hamiltonian form, and with the Weyl node located at $(p, q, k)=\left(0,0,0.5 k_{0}\right)$ carries a "charge" of -1 according to the usual definition. The topological charge of this Weyl point can also be numerically verified, using the method in Ref. [5] to calculate the Weyl point charge. As shown in the inset in Fig. 1(d), the Berry phases are defined on a spherical surface with a fixed azimuthal angle $\theta$ (red circle). We then track the evolution of the Berry phases as a function of $\theta$, as shown in Fig. 1(d), which shows that the Chern number of the band below the Weyl point decreases by one as $\theta$ increases from 0 to $\pi$. This implies that the Weyl point has a negative charge. (See Appendix B for more details.) 
(a)

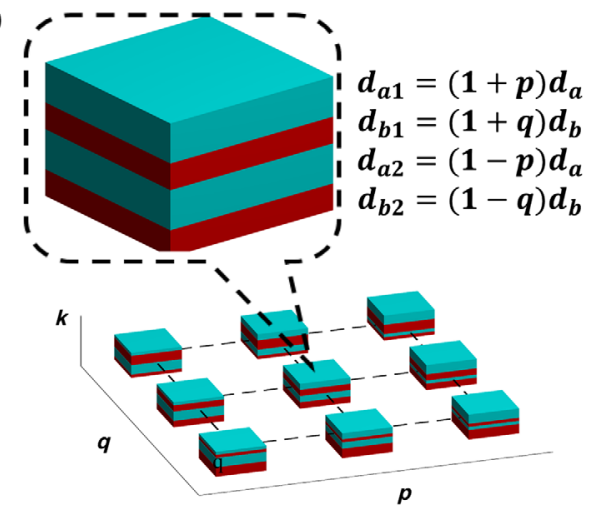

(c)

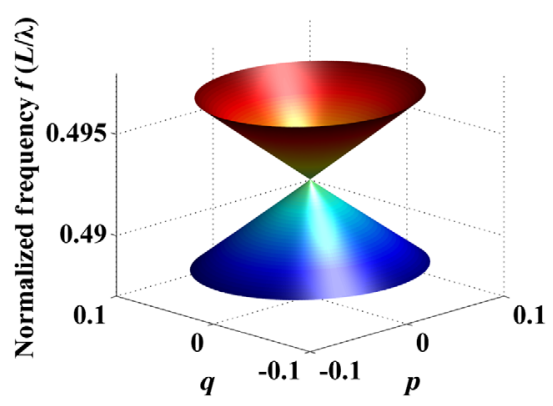

(b)

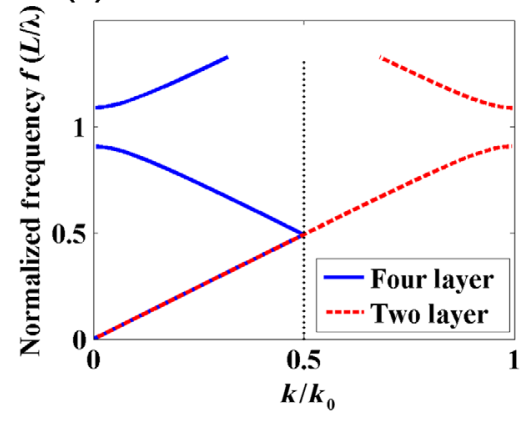

(d)

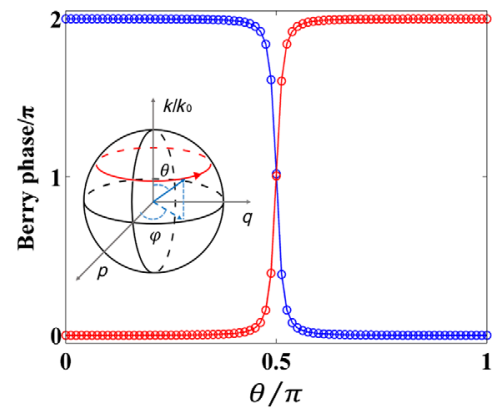

FIG. 1. Realization of Weyl points in a parameter space. (a) Photonic crystals (PCs) with different $p, q$ values. The $p, q$ form a parameter space. The inset shows one unit cell of the PC, where the first and the third layers are made of $\mathrm{H}_{\mathrm{f}} \mathrm{O}_{2}$ (blue), and the second and the fourth layers are made of $\mathrm{S}_{\mathrm{i}} \mathrm{O}_{2}$ (red). The thickness of each layer depends on its position in the $p$ - $q$ parameter space. (b) The band dispersion of PCs with different unit cells (red line for a PC with only two layers and blue line for a PC the unit cell of which consists of two unit cells used for the red line). Here, $d_{a}=97 \mathrm{~nm}$ and $d_{b}=72 \mathrm{~nm}$. (c) The dispersion of PCs in the $p-q$ space with $k=0.5 k_{0}$, and $k_{0}=\pi /\left(d_{a}+d_{b}\right)$. Here, two bands form a conical intersection. Panels (b) and (c) together show that the band dispersions are linear in all directions around the degeneracy point, indicating that it is an analogue of the Weyl point. (d) Berry phases defined on the spherical surface with a fixed $\theta$ (see the inset), where blue and red represent Berry phases on the lower and the upper band, respectively. The radius of the sphere is 0.001 .

In addition to the Weyl point constructed above, we also find Weyl points on higher bands and at different positions in the parameter space. In Fig. 2, we show all the Weyl points on the lower five bands at either $k=0.5 k_{0}$ or $k=0$. The locations and charges of these Weyl points are marked in the insets. The Weyl point between band 1, the lowest band, and band 2 [Fig. 2(a)] with charge -1 has already been discussed above. Two Weyl points with charge -1 exist between band 2 and band 3 . Weyl points with charge +1 appear on higher bands. To realize a Weyl point in the reciprocal space, one needs to break either inversion symmetry or time-reversal symmetry. Our systems possess time-reversal symmetry but do not exhibit inversion symmetry for general $p$ and $q$. Time-reversal symmetry requires that the charges of two Weyl points at $\left(p_{0}, q_{0}, k_{0}\right)$ and $\left(p_{0}, q_{0},-k_{0}\right)$ are the same. Besides time-reversal symmetry, our systems also exhibit the symmetry $\varepsilon(p, q, x)=\varepsilon(-p, q,-x)=\varepsilon(p,-q,-x)=\varepsilon(-p,-q, x)$, where $\varepsilon$ is the permittivity and $x$ represents the real space position. This symmetry ensures that as long as there is a Weyl point at $\left(p_{0}, q_{0}\right)$ at a particular frequency, there will be other Weyl points at $\left(-p_{0}, q_{0}\right),\left(p_{0},-q_{0}\right)$, and $\left(-p_{0},-q_{0}\right)$, and these Weyl points will all possess the same topological charge. Note that while the total charges of Weyl points must vanish in periodic systems [47] as the reciprocal space is periodic, such a constraint does not apply here as the parameter space is not periodic. This features one of the main differences between Weyl points in synthetic dimensions and those in momentum space.

\section{B. Reflection phases around the Weyl points}

We now consider the reflection phase of a normal incident plane wave when the PC is semi-infinite. The working frequency of the incident wave is chosen to be the frequency of the Weyl point between band 1 and band 2 . Except for the $p=q=0$ point, the working frequency is inside the band gap for all other $p$ and $q$ values. Hence, the reflection coefficient can be written as $r=\exp (i \phi)$, with $\phi$ being a function of $p$ and $q$. In Fig. 3(a), we show the reflection phase in the whole $p-q$ space, where the truncation boundary is at the center of the first layer. The reflection phase distribution shows a vortex structure, with the Weyl point at the vortex center. The topological 

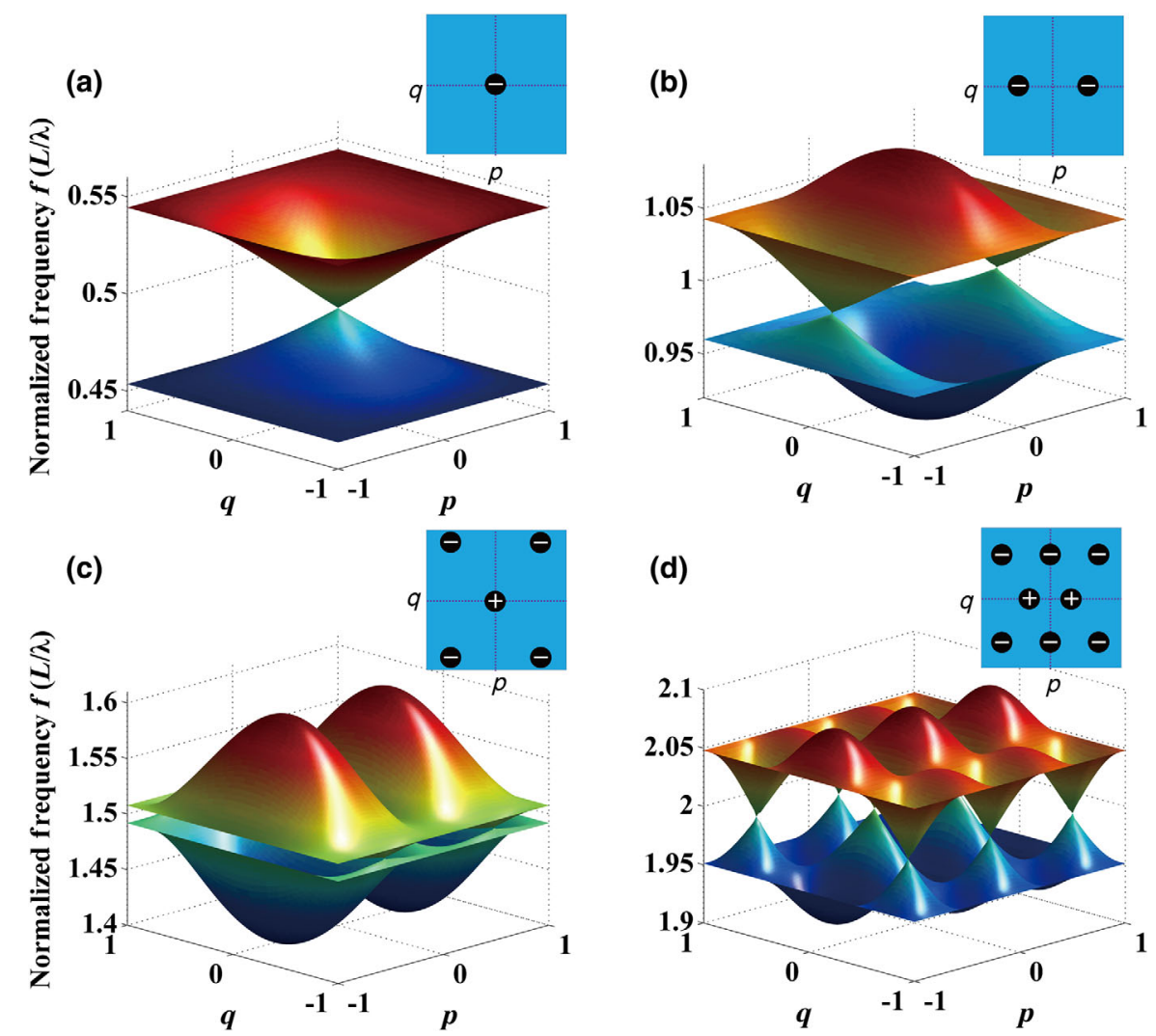

FIG. 2. The band dispersions in the parameter space at different $\mathrm{k}$ points. (a) Bands 1 and 2 at $k=0.5 k_{0}$. (b) Bands 2 and 3 at $k=0$. (c) Bands 3 and 4 at $k=0.5 k_{0}$. (d) Bands 4 and 5 at $k=0$. The conical intersections in (a)-(d) all correspond to Weyl points with positions and charges ("+" for charge +1 , "-" for charge -1 ) marked in the insets, in which the dashed lines denote $p=0$ or $q=0$. Here, we use the same parameters as those in Fig. 1.

charge of this vortex is given by the winding number of the phase gradient [48], which is the same as that of the Weyl point. For illustration purposes, we choose a circle centered at this Weyl point as marked by the gray dashed circle in Fig. 3(a). The reflection phase along the circular loop decreases with the polar angle $\varphi$, and picks up a total change of $-2 \pi$ after each circling. We note that the same circle does not enclose any Weyl points between band 2 and band 3. The reflection phase at the frequency of the Weyl points between band 2 and band 3 is shown in Fig. 3(c) (the circular loop is also marked). Now the reflection phase along the loop in Fig. 3(c) covers a range much less than $2 \pi$.

The above conclusion can also be experimentally verified. We choose five PC configurations whose locations in the $p-q$ space are indicated by the white circle, triangle, square, diamond, and pentagram in Figs. 3(a) and 3(c). The PCs here are fabricated by electron beam evaporation on a substrate made of K9 glass. Before evaporation, we clean the substrate with an acidic solution. During the evaporation, the pressure in the chamber is kept below $2 \times 10^{-3} \mathrm{~Pa}$, and the temperature is maintained at $90{ }^{\circ} \mathrm{C}$. The uncertainty in the thickness of each layer in the fabrication is below $10 \mathrm{~nm}$ for all the PCs. Here, we set $d_{a}=97 \mathrm{~nm}$, $d_{b}=72 \mathrm{~nm}$, and the number of unit cells to 15 . The layers in these five PCs are given by $(p, q)=$ $(0.24,0.44),(-0.11,0.49),(-0.38,0.31),(-0.45,0.22)$, and $(0.33,-0.37)$, respectively. We use two spectrometers to measure the reflection phase of the PCs. One spectrometer ranges from 880 to $1700 \mathrm{~nm}$ with a resolution of $0.8 \mathrm{~nm}$ (BWTEK-BTC 261E Cooled InGaAs Array Spectrometer). The other ranges from 330 to $1070 \mathrm{~nm}$ with a resolution of $0.8 \mathrm{~nm}$ (BWTEK-BTC 611E Spectrometer). The reflection phase is measured using a Fabry-Perot interference setup (see Sec. I of the Supplemental Material for a more detailed setup [49]).

The measured reflection phases across the first and second band gaps are shown in Figs. 3(b) and 3(d), respectively, where different markers correspond to different samples. Black dashed vertical lines mark the frequency of the Weyl points and gray areas represent the passband region. We also numerically calculate the reflection phases, and we show the results in Figs. 3(b) and 3(d) with colored curves. The experimental results agree reasonably well with the numerical results. The reflection phases in Fig. 3(b) are uniformly distributed and cover the whole $[-\pi \pi)$ range, while the reflection phases in Fig. 3(d) cover only a narrow range. Whether the reflection phase trajectories span the 
(a)

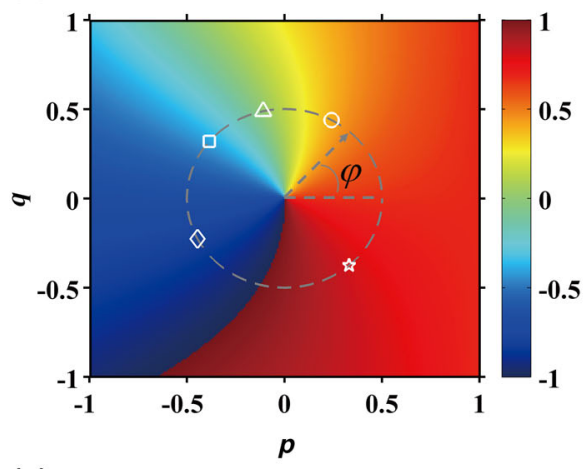

(c)

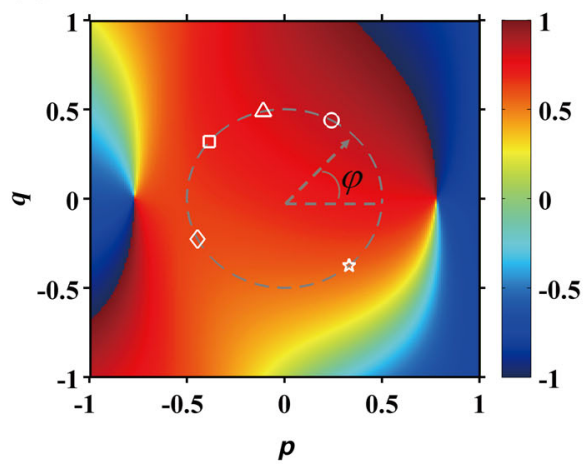

(b)

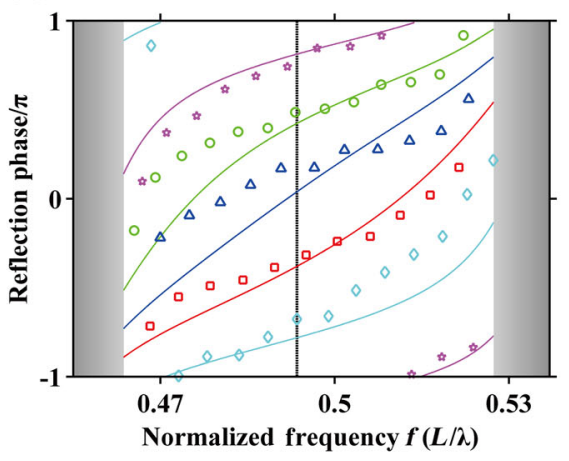

(d)

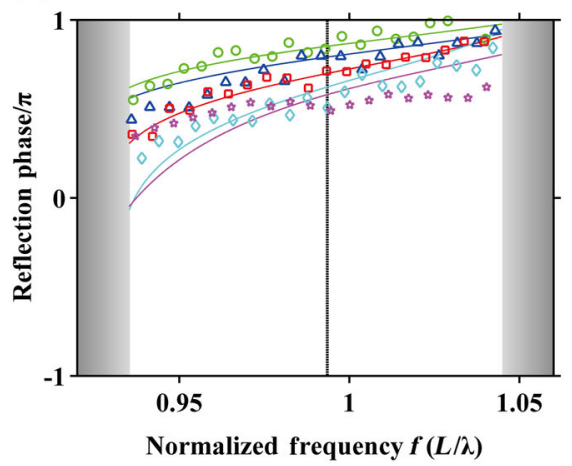

FIG. 3. The reflection phase in the $p-q$ space. (a),(c) Reflection phase in the $p-q$ space at the frequency of the Weyl point in Figs. 2(a) and 2(b). The reflection phase shows a vortex structure with charge -1 around Weyl points. The white circle, triangle, square, diamond, and pentagram mark the values of $(p, q)$ of the five samples, which are, respectively, $(p, q)=(0.24,0.44)$, $(-0.11,0.49),(-0.38,0.31),(-0.45,0.22)$, and $(0.33,-0.37)$. These five samples have configurations located on a circle (dashed gray line) in the parameter space which encloses a Weyl point in (a) but does not enclose any Weyl point in (c). (b),(d) Reflection phases in the band gaps of the five samples in (a) and (c). The markers show the experimental results whose shape marks the position in the $p-q$ space in (a) and (c), and colored lines represent the corresponding reflection phases from numerical simulations. The black dashed lines mark the frequencies of the Weyl points in (a) and (c), and the bulk band regions of the PC with the narrowest band gap are shaded in gray.

gap region [as in Fig. 3(b)] or not [as in Fig. 3(d)] depends on whether the loop in the parameter space encloses a Weyl node or not, given the fact that the total phase change must be $2 \pi$ if the loop encircles a Weyl point; a proof is in Appendix C. We emphasize that the vortex structure and the charge of the vortex are independent of the position where we truncate the PCs. Though the reflection phase vortex we discuss here is in the generalized parameter space, the reflection phase vortex acts as a signature of Weyl physics. See, for example, Ref. [45] and the discussions in Appendix C.

\section{Fermi-arc-like interface states}

The vortex structure and the associated topological charge guarantee the existence of interface states [30,31] between the PCs with Weyl points and the reflecting substrates, regardless of the substrate properties. The existence of interface states [30] is given by

$$
\phi_{\mathrm{PC}}+\phi_{S}=2 m \pi, \quad m \in \mathbb{Z},
$$

where $\phi_{\mathrm{PC}}$ and $\phi_{S}$ represent the reflection phases of the $\mathrm{PC}$ and the reflecting substrate, respectively. As the reflection phase on the loop encircling the Weyl point covers the whole $[-\pi, \pi)$ region, no matter what the reflection phase of the reflecting substrate is, Eq. (3) can always be satisfied for at least one polar angle. If we consider loops with different radii, then the interface states form a continuous trajectory beginning from the Weyl point. The trajectory of the interface states ends either at another Weyl point with an opposite charge or at the boundary of the parameter space. The behavior of these interface states connecting Weyl points with opposite charges in the parameter space has the same mathematical origin as that of the Fermi arc $[1,2,4,7]$ in Weyl semimetals. There is, however, a crucial difference: the Fermi arc starts and ends with Weyl points in a periodic system, while the interface states in our system can connect Weyl points to the boundary of the parameter space because the total charge of the Weyl points does not vanish inside the parameter space. 
(a)

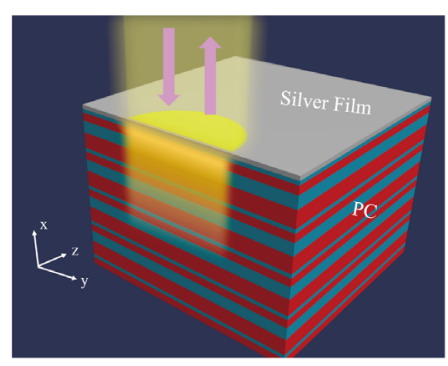

(b)

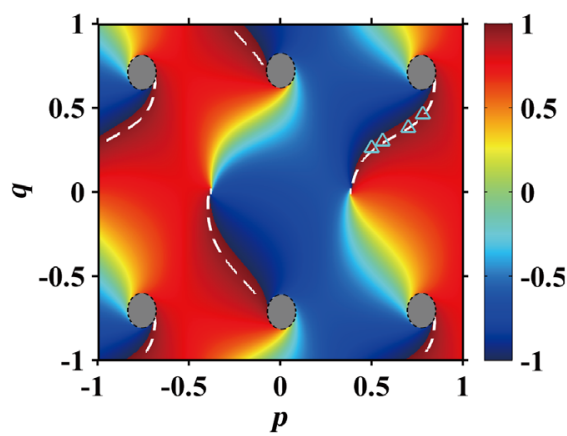

(c)

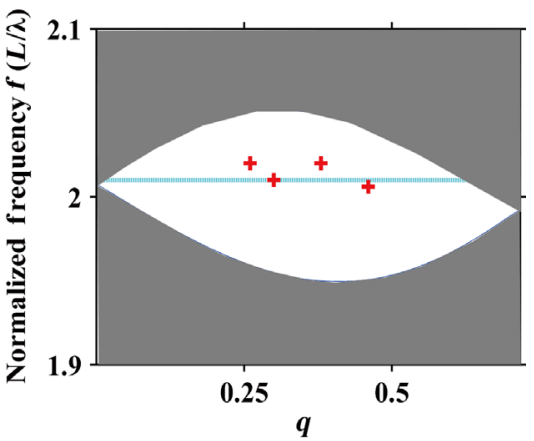

FIG. 4. The "Fermi arc" in the parameter space. (a) A sketch shows the structure used in measuring the interface states. Here, the silver slab represents the silver film, blue represents $\mathrm{H}_{f} \mathrm{O}_{2}$, and red represents $\mathrm{S}_{i} \mathrm{O}_{2}$. Wave incidents from the silver film side and the interface states are localized in the interface region between the silver slab and the semi-infinite PC. The amplitude of the wave is shown schematically in yellow. (b) The reflection phase of the PCs at the frequency of Weyl points with charge +1 with its dispersion shown in Fig. 2(d). The bulk band regions at the working frequency are shaded in gray. The white dashed lines show the trajectories of the interface states of a system consisting of a semi-infinite PC coated with a silver film, where the semi-infinite PC is truncated at the center of the first layer. These interface state trajectories are analogues of Fermi arc states. The triangles mark the $p$ and $q$ values of the four samples in the experiment. (c) The cyan line indicates the working frequency used in (b), and the red crosses label the experimental results. The projection of the bulk band as a function of $q$ is shaded in gray. The number of unit cells is 10 , and $d_{a}=0.323 \mu \mathrm{m}$ and $d_{b}=0.240 \mu \mathrm{m}$ for the four PCs in (c). The thickness of layers in these four PCs are given by $(p, q)=(0.50,0.26),(0.56,0.30)$, $(0.70,0.38)$, and $(0.78,0.36)$, respectively.

As an example, we consider the Weyl points on the fourth and fifth bands as shown in Fig. 2(d). There are a total of eight Weyl points: six with charge -1 and the remaining two with charge +1 . As our system does not possess any external symmetry that can relate two Weyl points with opposite charges, the frequency of the Weyl points with a positive charge is higher than that of the Weyl points with a negative charge. To turn the Weyl points into the working frequency range of our measurements, we increase the thickness of each slab in the PCs proportionally and we now have $d_{a}=0.323 \mu \mathrm{m}$ and $d_{b}=0.240 \mu \mathrm{m}$. We then set the working frequency to the frequency of the Weyl point with a positive charge $(303 \mathrm{THz})$ and truncate the PCs at the center of the first layer. A sketch of measuring the interface states is shown in Fig. 4(a). Here, the silver slab represents the silver film, blue represents $\mathrm{H}_{\mathrm{f}} \mathrm{O}_{2}$, and red represents $\mathrm{S}_{\mathrm{i}} \mathrm{O}_{2}$. Wave incidents from the silver film side and the interface states are localized in the interface region between the silver slab and the semi-infinite PC. The amplitude of the wave is shown schematically in yellow. In Fig. 4(b), we show the reflection phase in the parameter space, where gray areas within black dashed lines mark the regions of the bulk band. If the truncated PCs are coated with silver films, then interface states exist that satisfy Eq. (3). The reflection phase of the silver film coated on the PCs is measured to be $(-0.95 \pm 0.0471) \pi$ at $303 \mathrm{THz}$. The white dashed lines in Fig. 4(b) show the trajectories of interface states. In addition to interface states connecting Weyl points with different charges, there are also trajectories of interface states terminating at the boundary of our parameter space. We also perform experiments to verify the results. We choose four points [positions marked with cyan triangles in Fig. 4(b)] to experimentally demonstrate the existence of the interface states, with samples consisting of 10 periods, and the thickness of the layers in these four PCs are given by $(p, q)=(0.50,0.26),(0.56,0.30),(0.70,0.38)$, and $(0.78,0.36)$ respectively. The results are shown in Fig. 4(c) (red crosses) and match well with the numerical prediction (cyan dashed line); a more detailed setup can be found in Sec. II of the Supplemental Material [49]. We emphasize here that the existence of interface states is "robust" to the property of the reflecting substrates: we can always find trajectories of interface states that link the two Weyl points with opposite charges no matter what the reflecting substrate is (See Sec. III of the Supplemental Material [49]).

\section{D. "Nodal lines" in higher-dimensional space}

Compared with Bloch momentum space, synthetic dimensions provide a flexible way to construct topological systems in higher-dimensional space, which enables the study of phenomena that occur only in higher-dimensional spaces [23]. As an example, we can define another parameter $R$ as the ratio $R=n_{a} d_{a} /\left(n_{a} d_{a}+n_{b} d_{b}\right)$, which belongs to $(0,1)$. By varying this parameter, we can now observe topological transitions of the band structures. In Fig. 5, we show the dispersion of the fourth and fifth band gaps with $k=0$ for different values of $R$. The insets show the positions of the Weyl points (black circles) and degenerate lines (dashed lines). When $R=0.25$ [Fig. 5(c)], 0.5 [Fig. 5(e)], and 0.75 [Fig. 5(g)], only degenerate lines exist, which act as analogues of the nodal lines in semimetals [50]. When $R$ passes through these transition 

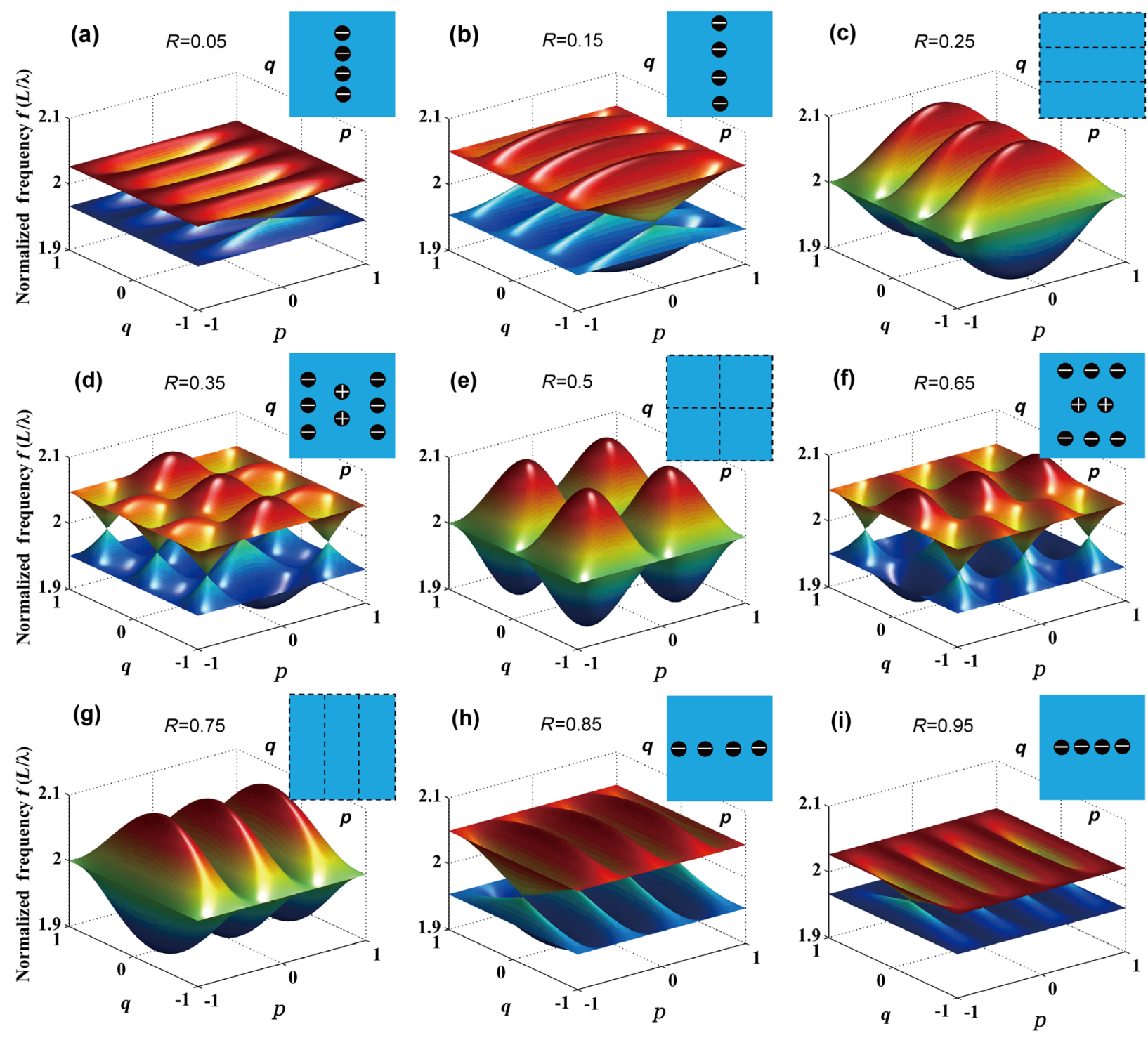

FIG. 5. Topological transitions occur as we change the parameter $R$ defined in the text. (a)-(i) Band structures of band 4 and band 5 with different values of $R$ at $k=0$. The values of $R$ are $0.05,0.15,0.25,0.35,0.5,0.65,0.75,0.85$, and 0.95 for (a)-(i), respectively. The insets show the positions of the Weyl points ("+" for positive charge and "-" for negative charge) and "nodal lines" (black dashed lines).

points, Weyl points will immerse as node lines and reappear. In the process, a pair of Weyl point with positive and negative charge will appear or disappear simultaneously. Although the number of Weyl points changes, the total charge remains constant.

\section{CONCLUSION}

We show the existence of Weyl points in the parameter space and their topological consequences. In the specific example of dielectric superlattices, the reflection phase of the semi-infinite multilayered PC shows a vortex structure with the same topological charge as the synthetic Weyl points defined in the parameter space. The vortex structure guarantees the existence of interface states, which can be used in various systems [33-42]. (See also one example given in Sec. IV of the Supplemental Material [49].) In general, interface states may or may not exist at the boundary between a 1D PC and a reflecting substrate [30]. The Weyl points here provide a deterministic scheme to construct interface states between multilayered PCs and reflecting substrates of arbitrary reflection phases [31]. In the past, numerical simulation was the only way to predict the optical properties of photonic crystals with complex unit cells defined by many parameters and there was no easy way to connect the bulk properties to the surface properties such as reflectance and the existence of interface states. Here, we see that the topological character of the nodal points in the higher-dimension space defined jointly by momentum and structural parameters actually connects 
the properties of the bulk to those of the surface. We emphasize that the topological notions apply to all frequencies, including high-frequency gaps where the PCs cannot be described by the effective medium theory.

The geometric parameters $p$ and $q$ are fixed for each $\mathrm{PC}$ in this work. However, there are techniques [51-54] that can be employed to control the geometric parameters as well as the refractive index in real time. Combined with the understanding of the topological origin of the interface states, such tunability allows for the control of the interface states, which may facilitate various applications. In addition, the reflection phase vortex offers a flexible way to manipulate the electromagnetic wave, such as generating vortex beams and controlling the reflection direction. This work also opens up a new direction for experimentally exploring the physics in topological theory in higher dimensions [23]. With more parameters involved, we can also construct Weyl points with a higher topological charge $[12,13,55]$.

\section{ACKNOWLEDGMENTS}

H. L. thanks Xian Gang Wan for helpful discussions. H. L. gratefully acknowledges the support of the National Key Projects for Basic Researches of China (Grants No. 2017YFA0205700 and No. 2017YFA0303700), and the National Natural Science Foundation of China (Grants No. 11690033, No. 61425018, No. 11621091, and No. 11374151). C. T. C. and M. X. gratefully acknowledge the support of the Research Grants Council, Hong Kong (AoE/P-02/12).

Q. W. and M.X. proposed and designed the system. Q. W., H. L., and S. Z. carried out the experiments. Q. W., M. X., C. T. C., and H. L. contributed to the experimental characterization and interpretation and developed the theory. Q. W., M. X., and C. T. C. co-wrote the manuscript. All of the authors were involved in the discussions. Q. W. and $\mathrm{M}$. X. contributed equally to this work.

\section{APPENDIX A: EFFECTIVE HAMILTONIAN AROUND THE WEYLD POINTS}

Here, we adapt the transfer matrix method to derive the effective Hamilton around the Weyl points. Each unit cell is composed of four layers, the transfer matrix can be written as

$$
\mathbf{T}=\left(\begin{array}{cc}
C & D \\
D^{*} & C^{*}
\end{array}\right)\left(\begin{array}{cc}
A & B \\
B^{*} & A^{*}
\end{array}\right)
$$

where

$$
\begin{gathered}
A=e^{i k_{a} d_{a 1}}\left[\cos \left(k_{b} d_{b 1}\right)+\frac{i}{2}\left(\frac{n_{b}}{n_{a}}+\frac{n_{a}}{n_{b}}\right) \sin \left(k_{b} d_{b 1}\right)\right], \\
B=e^{-i k_{a} d_{a 1}}\left[\frac{i}{2}\left(\frac{n_{b}}{n_{a}}-\frac{n_{a}}{n_{b}}\right) \sin \left(k_{b} d_{b 1}\right)\right], \\
C=e^{i k_{a} d_{a 2}}\left[\cos \left(k_{b} d_{b 2}\right)+\frac{i}{2}\left(\frac{n_{b}}{n_{a}}+\frac{n_{a}}{n_{b}}\right) \sin \left(k_{b} d_{b 2}\right)\right], \\
D=e^{-i k_{a} d_{a 2}}\left[\frac{i}{2}\left(\frac{n_{b}}{n_{a}}-\frac{n_{a}}{n_{b}}\right) \sin \left(k_{b} d_{b 2}\right)\right],
\end{gathered}
$$

and the wave equation can be written as

$$
\left[\mathbf{T}-e^{i 2 k\left(d_{a}+d_{b}\right)}\right]\left(\begin{array}{c}
c^{+} \\
c^{-}
\end{array}\right)=0
$$

where $c^{+}$and $c^{-}$represent the coefficients of the forwardpropagating and backward-propagating waves inside the first layer, respectively. We define the following dimensionless coefficients:

$$
\begin{aligned}
& \xi_{f}=\left(f-f_{w}\right) / f_{w}, \\
& \xi_{p}=\left(p-p_{w}\right), \\
& \xi_{q}=\left(q-q_{w}\right), \\
& \xi_{k}=\left(k-k_{w}\right) / k_{0} .
\end{aligned}
$$

Here, $\left(p_{w}, q_{w}, k_{w}\right)$ denotes the position of the Weyl point under consideration, and $f_{w}$ is the frequency of the Weyl point. The system under consideration supports only one forward-propagating wave and one backward-propagating wave. Hence, if there is a Weyl point, the Weyl point must be located at the Brillouin zone center or zone boundary. Expanding $\mathbf{T}$ with respect to $\left(\xi_{p}, \xi_{q}, \xi_{f}\right)$ around the Weyl point, it is easy to show that the zero-order term of $\mathbf{T}$ is either 1 or -1 (depending on whether the Weyl point is at the zone boundary or the zone center). Hence, up to the first order of $\left(\xi_{p}, \xi_{q}, \xi_{f}\right)$, we have

$$
\mathbf{T}=\left(\begin{array}{cc} 
\pm 1+i c_{1} \xi_{p}+i c_{2} \xi_{q}+i c_{3} \xi_{f} & d_{1} \xi_{p}+d_{2} \xi_{q}+d_{3} \xi_{f} \\
d_{1}^{*} \xi_{p}+d_{2}^{*} \xi_{q}+d_{3}^{*} \xi_{f} & \pm 1-\left(i c_{1} \xi_{p}+i c_{2} \xi_{q}+i c_{3} \xi_{f}\right)
\end{array}\right)+O\left(\xi_{p}^{2}, \xi_{q}^{2}, \xi_{f}^{2}\right)
$$

Meanwhile, as $\operatorname{det}(\mathbf{T})=1$, one can prove that $\left\{c_{1}, c_{2}, c_{3}\right\} \in \mathbb{R}$. Keeping only the first order, Eq. (A6) can be written as

$$
\left(\begin{array}{cc}
-\left(c_{1} \xi_{p}+c_{2} \xi_{q}-c_{4} \xi_{k}\right) & -i\left(d_{1} \xi_{p}+d_{2} \xi_{q}\right) \\
i\left(d_{1}^{*} \xi_{p}+d_{2}^{*} \xi_{q}\right) & -\left(c_{1} \xi_{p}+c_{2} \xi_{q}+c_{4} \xi_{k}\right)
\end{array}\right)\left(\begin{array}{c}
-i c^{+} \\
i c^{-}
\end{array}\right)=\xi_{f}\left(\begin{array}{cc}
c_{3} & i d_{3} \\
-i d_{3}^{*} & c_{3}
\end{array}\right)\left(\begin{array}{c}
-i c^{+} \\
i c^{-}
\end{array}\right),
$$


(a)

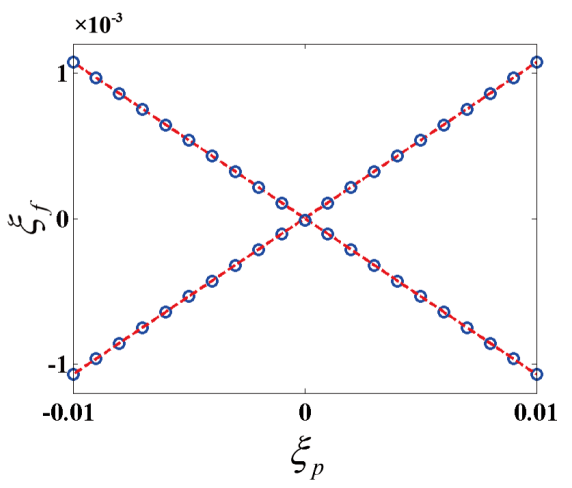

(b)

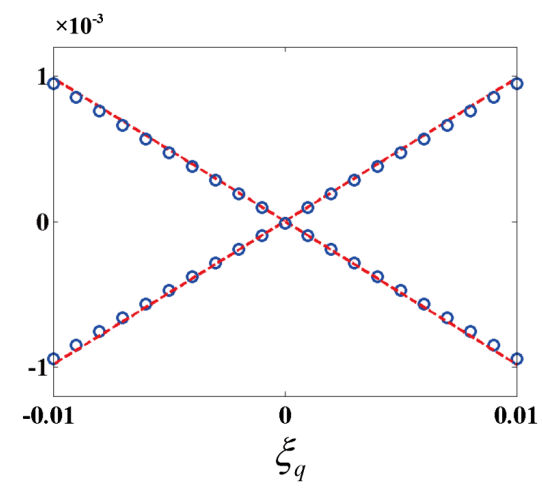

(c)

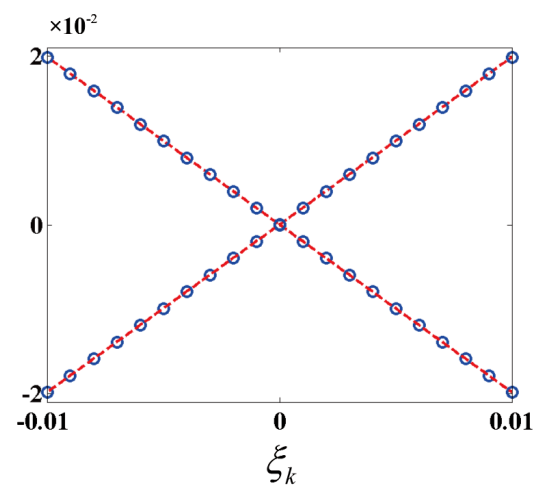

FIG. 6. The dispersion near the Weyl points. Panels (a)-(c) show the comparison between the numerical result (blue circles) and the effective Hamiltonian in Eq. (A16) (red dashed lines) in three directions. The parameters of the PC are given by $d_{a}=97 \mathrm{~nm}$, $d_{b}=72 \mathrm{~nm}$, and the Weyl point considered is between the first and the second bands.

where $\left\{c_{1}, c_{2}, c_{3}, c_{4}\right\} \in \mathbb{R}$ and $\left\{d_{1}, d_{2}, d_{3}\right\} \in \mathbb{C}$. Note that now the matrixes on both sides of Eq. (A9) are Hermitian matrixes. The matrix on the right-hand side of Eq. (A9) can be decomposed as

$$
\left(\begin{array}{cc}
c_{3} & i d_{3} \\
-i d_{3}^{*} & c_{3}
\end{array}\right)=U\left(\begin{array}{cc}
c_{3}-\left|d_{3}\right| & 0 \\
0 & c_{3}+\left|d_{3}\right|
\end{array}\right) U^{\dagger},
$$

where

$$
U=\frac{1}{\sqrt{2}}\left(\begin{array}{cc}
-i d_{3} /\left|d_{3}\right| & i d_{3} /\left|d_{3}\right| \\
1 & 1
\end{array}\right)
$$

After some simple mathematics, Eq. (A9) can be written as

$$
\begin{aligned}
& \left(\begin{array}{cc}
c_{3}-\left|d_{3}\right| & 0 \\
0 & c_{3}+\left|d_{3}\right|
\end{array}\right)^{-1 / 2} U^{\dagger} M U\left(\begin{array}{cc}
c_{3}-\left|d_{3}\right| & 0 \\
0 & c_{3}+\left|d_{3}\right|
\end{array}\right)^{-1 / 2} \\
& \times\left(\begin{array}{c}
-i \tilde{c}^{+} \\
i \tilde{c}^{-}
\end{array}\right)=\xi_{f}\left(\begin{array}{c}
-i \tilde{c}^{+} \\
i \tilde{c}^{-}
\end{array}\right),
\end{aligned}
$$

where

$M=\left(\begin{array}{cc}-\left(c_{1} \xi_{p}+c_{2} \xi_{q}-c_{4} \xi_{k}\right) & -i\left(d_{1} \xi_{p}+d_{2} \xi_{q}\right) \\ i\left(d_{1}^{*} \xi_{p}+d_{2}^{*} \xi_{q}\right) & -\left(c_{1} \xi_{p}+c_{2} \xi_{q}+c_{4} \xi_{k}\right)\end{array}\right)$,

and

$$
\left(\begin{array}{c}
-i \tilde{c}^{+} \\
i \tilde{c}^{-}
\end{array}\right)=\left(\begin{array}{cc}
c_{3}-\left|d_{3}\right| & 0 \\
0 & c_{3}+\left|d_{3}\right|
\end{array}\right)^{1 / 2} U^{\dagger}\left(\begin{array}{c}
-i c^{+} \\
i c^{-}
\end{array}\right) .
$$

Equation (A12) can be reformulated in the Pauli matrix form as

$$
\left(\begin{array}{lll}
\xi_{p} & \xi_{q} & \xi_{k}
\end{array}\right) \mathbf{v}\left(\begin{array}{c}
\sigma_{0} \\
\sigma_{x} \\
\sigma_{y} \\
\sigma_{z}
\end{array}\right)\left(\begin{array}{c}
-i \tilde{c}^{+} \\
i \tilde{c}^{-}
\end{array}\right)=\xi_{f}\left(\begin{array}{c}
-i \tilde{c}^{+} \\
i \tilde{c}^{-}
\end{array}\right),
$$

where $\sigma_{i}(i=x, y, z)$ is the Pauli matrix, $\sigma_{0}$ is a $2 \times 2$ identity matrix, and $\mathbf{v}$ is a $3 \times 4$ real matrix. Hence, the matrix on the left-hand side of Eq. (A12),

$$
H \equiv\left(\begin{array}{lll}
\xi_{p} & \xi_{q} & \xi_{k}
\end{array}\right) \mathbf{v}\left(\begin{array}{c}
\sigma_{x} \\
\sigma_{y} \\
\sigma_{z} \\
\sigma_{0}
\end{array}\right),
$$

works as an effective Hamiltonian of the system. This effective Hamiltonian processes a Weyl form.

We choose the Weyl point between band 1 and band 2 as an example. Here, $\left(p_{w}, q_{w}, k_{w}\right)=\left(0,0,0.5 k_{0}\right)$, with $k_{0}=\pi /\left(d_{a}+d_{b}\right)$. For the PC with $d_{a}=97 \mathrm{~nm}$, $d_{b}=72 \mathrm{~nm}$, the frequency of this Weyl point is at $f_{w}=247.6 \mathrm{THz}$. Around this Weyl point, the coefficient matrix is given by

$$
\mathbf{v}=\left(\begin{array}{cccc}
0 & 0 & 0.1073 & 0 \\
0 & -0.0946 & 0 & 0 \\
-1.985 & 0 & 0 & 0
\end{array}\right)
$$

Hence, according to the definition, the charge of this Weyl point is -1 . For other Weyl points, there are more nonzero coefficients in Eq. (A17). To verify that the obtained effective Hamiltonian works, we plot the dispersion along the three directions $p, q, k$ in Figs. 6(a)-6(c), respectively. Red dashed lines are calculated using the effective Hamiltonian, while the blue circles are from the full wave simulations. They agree well near the Weyl point, which shows that the effective Hamiltonian works. 


\section{APPENDIX B: NUMERICAL CALCULATIONS OF THE TOPOLOGICAL CHARGES}

We adapt a numerical method used in the literature to determine the charges of Weyl points. We first calculate the Berry phase on the circle on a spherical surface (centered at the Weyl point) with a fixed $k$, and then trace the evolutions of the Berry phases as $k$ varying from the upper pole to the lower pole. The total change of the Berry phase corresponds to the Chern number of this spherical surface. To illustrate this idea, we choose two loops $l_{i}$ and $l_{i+1}$, as shown in Fig. 7(a), and assume the Berry phases of them are given by $\gamma_{i}$ and $\gamma_{i+1}$, respectively. The Berry phases along these loops equal the Berry fluxes out of the upper spherical crown of these two loops. The Berry phases here are well defined up to an uncertainty of $2 m \pi(\mathrm{m} \in \mathbb{Z})$. When $l_{i}$ and $l_{i+1}$ are very close to each other, the Berry phases of these two loops must also be close to each other and the difference between them equals the Berry flux coming out from the strip region between $l_{i}$ and $l_{i+1}$. Hence, the change of the Berry phase over the whole spherical surface equals the Berry flux out of the Weyl point.

To numerically calculate the Berry phase for each loop, we use a discretized algorithm [5]:

$\gamma_{n}=-\operatorname{Im} \sum_{i=1}^{N} \ln \left(\int_{0}^{L} \varepsilon(x) u_{n, p_{i}, q_{i}, k}^{*}(x) u_{n, p_{i+1}, q_{i+1}, k}(x) d x\right)$,

where $\varepsilon$ represents the permittivity and $u_{n, p_{i}, q_{i}, k}(x)$ is the periodic-in-cell part of the eigenelectric field of a state on the $n$th band and with parameters $p_{i}, q_{i}$, and $k$. Here, $p_{i}=r \sin (\theta) \cos (i 2 \pi / N), q_{i}=r \sin (\theta) \sin (i 2 \pi / N)$, and $N$ has been chosen to be large enough such that $\gamma_{n}$ converges. (a)

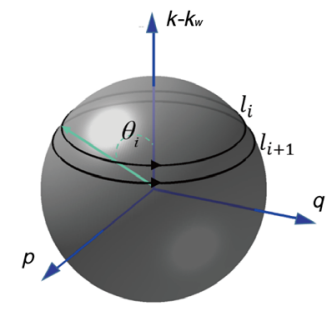

(b)

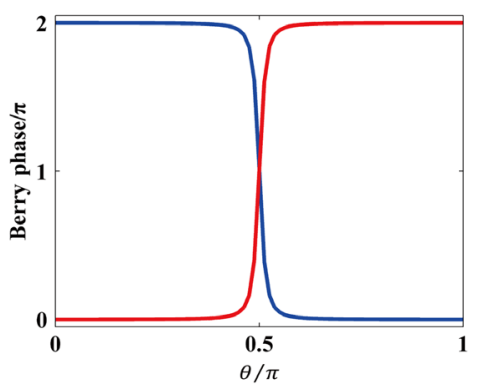

FIG. 7. The method to calculate the charge of the Weyl point numerically. (a) Schematic illustration of the integration paths used to calculate topological charges of Weyl points. (b) Berry phases defined on the spherical surface with fixed $\theta$, where blue and red represent Berry phases on the lower band and the upper band, respectively. The parameters of the PC are given by $d_{a}=97 \mathrm{~nm}, d_{b}=72 \mathrm{~nm}$, and the Weyl point considered is between the first and the second band. The radius of the sphere is 0.001 .
In Fig. 7(b), we plot Berry phases on band 1 (blue) and band 2 (red) as a function of $\theta$. In the calculation, the radius of the sphere in Fig. 7 is set to be $r=0.001$. We can see that the variation range of the Berry phase is $2 \pi$ for the upper band (the red line) and $-2 \pi$ for the lower band (the blue line), which means the charge of this Weyl point is -1 .

\section{APPENDIX C: REFLECTION PHASES AROUND WEYL POINTS}

In this Appendix, we show how to get the reflection phase from the effective Hamiltonian obtained in Appendix A. We choose the Weyl point between the first and second bands as an example. The Hamiltonian of our system near this Weyl point is given by

$$
H=\xi_{p} v_{p z} \sigma_{z}+\xi_{q} v_{q y} \sigma_{y}+\xi_{k} v_{k x} \sigma_{x},
$$

where $v_{p z}=0.1073, v_{q y}=-0.0946$, and $v_{k x}=-1.985$ for the Weyl point under consideration. Now let us consider an elliptical loop circling the Weyl point in the parameter space spanned by $\xi_{p}$ and $\xi_{q}$ :

$$
\begin{aligned}
& \xi_{p}\left|v_{p z}\right|=r \cos \left(\varphi^{\prime}\right), \\
& \xi_{q}\left|v_{q y}\right|=r \sin \left(\varphi^{\prime}\right),
\end{aligned}
$$

where $\varphi^{\prime}$ is the polar angle in the new parameter space and $\varphi^{\prime} \in[0,2 \pi) . \varphi^{\prime}$ processes a one-to-one correspondence to the polar angle in the original parameter space $\varphi$ through the relation

$$
\tan \left(\varphi^{\prime}\right)\left|v_{p z}\right|=\left|v_{q y}\right| \tan (\varphi),
$$

In the following, we obtain the reflection phase as a function of $\varphi^{\prime}$ and show that the charge of the vortex of the reflection phase is equal to the charge of the Weyl point. The elliptical loop is chosen as defined in Eq. (C2) to simplify the derivation. Here, we choose the working frequency to be inside the bulk band gap, and, hence, $\xi_{f}<r$. We define $\cos \chi=\left(\xi_{f} / r\right)$, with $\chi \in(0, \pi)$. Substituting Eq. (C2) into Eq. (C1), we obtain two solutions of $\xi_{k}$ as

$$
\xi_{k} v_{k x}= \pm i r \sin \chi
$$

As expected, the wave vector $\xi_{k}$ becomes purely imaginary. We assume that the direction of the incident wave is along the positive direction, and, hence, we require $\operatorname{Im}\left(\xi_{k}\right)>0$. The corresponding eigenstate is given by

$$
\vec{u}=\left(\begin{array}{c}
1 \\
-i \beta
\end{array}\right),
$$

where 


$$
\beta=-\frac{\cos (\chi)-\cos \left(\varphi^{\prime}\right)}{\sin (\chi)-\sin \left(\varphi^{\prime}\right)}=\tan \left[\left(\varphi^{\prime}+\chi\right) / 2\right] .
$$

$\phi=\pi+2 \arctan \left[-z_{a} \tan \left(\psi_{1} / 2+\psi_{2}+k_{a} x-\pi / 4\right)\right]$.

Substituting Eq. (C5) in to Eq. (A14), we obtain the coefficients of the forward-propagating and backwardpropagating wave as

$$
\left(\begin{array}{c}
c^{+} \\
c^{-}
\end{array}\right)=\frac{1}{\sqrt{2}}\left(\begin{array}{c}
e^{i \psi_{1}}\left[\left(c_{3}-\left|d_{3}\right|\right)^{-1 / 2}+i \beta\left(c_{3}+\left|d_{3}\right|\right)^{-1 / 2}\right] \\
-i\left[\left(c_{3}-\left|d_{3}\right|\right)^{-1 / 2}-i \beta\left(c_{3}+\left|d_{3}\right|\right)^{-1 / 2}\right]
\end{array}\right),
$$

where $c_{3}$ and $d_{3}$ are the coefficients defined in Eq. (A8) and $\psi_{1}=\arg \left(d_{3}\right)$. To simplify the notation, we define

$$
\psi_{2} \equiv \tan ^{-1}\left[\beta\left(\frac{c_{3}-\left|d_{3}\right|}{c_{3}+\left|d_{3}\right|}\right)^{1 / 2}\right]
$$

and

$$
A_{0}=\sqrt{\frac{1}{c_{3}-\left|d_{3}\right|}+\frac{\beta^{2}}{\left(c_{3}+\left|d_{3}\right|\right)}} .
$$

Now Eq. (C7) can be simplified as

$$
\left(\begin{array}{c}
c^{+} \\
c^{-}
\end{array}\right)=A_{0} \sqrt{\frac{1}{2}}\left(\begin{array}{c}
e^{i \psi_{1}} e^{i \psi_{2}} \\
-e^{i \pi / 2} e^{-i \psi_{2}}
\end{array}\right)
$$

Then the corresponding electronic field at the boundary of the $\mathrm{PC}$ is

$$
\begin{aligned}
E_{1 y} & =c^{+} e^{i k_{a} x}+c^{-} e^{-i k_{a} x} \\
& =A_{0}\left[\left(e^{i \psi_{1}} e^{i \psi_{2}}\right) e^{i k_{a} x}-\left(e^{i \pi / 2} e^{-i \psi_{2}}\right) e^{-i k_{a} x}\right] / \sqrt{2},
\end{aligned}
$$

where the subscript " $1 y$ "means the electric field is along the $y$ direction and inside the first layer, $k_{a}$ represents the wave vector in the first layer, and $x$ represents the distance from the truncated plane to the starting plane of the first layer. The corresponding magnetic field is

$$
H_{1 z}=\frac{1}{\sqrt{2} z_{a}} A_{0}\left[\left(e^{i \psi_{1}} e^{i \psi_{2}}\right) e^{i k_{a} x}+\left(e^{i \pi / 2} e^{-i \psi_{2}}\right) e^{-i k_{a} x}\right],
$$

where $z_{a}$ is the bulk impedance of the first layer (in units of the vacuum impedance $z_{0}$ ). Thus, the surface impedance of the photonic crystal can be written as

$$
Z_{p c}=i z_{a}\left[\tan \left(\psi_{1} / 2+\psi_{2}+k_{a} x-\pi / 4\right)\right],
$$

and the corresponding reflection phase as
To show the validity of this method, we compare the reflection phase obtained from Eq. (C14) to that from the full wave simulation (where we use the transfer matrix method). As the working frequency is inside the band gap and if the number of unit cells is large enough, the reflection phase will converge. In the full wave simulation, we ensure that the number of unit cells is large enough. In Fig. 8, the red circles and blue solid line represent the reflection phases obtained with Eq. (C14) and the transfer matrix method, respectively. They agree quite well with each other, which shows that Eq. (C14) works in the region near the Weyl point.

Now let us continue to analyze the monotonicity and dependence of the reflection phase obtained in Eq. (C14). It is easy to see that $\phi$ is a monotonically decreasing function of $\psi_{1} / 2+\psi_{2}+k_{a} x-\pi / 4$. The dependences of $\phi$ on the polar angle $\varphi$ and the working frequency are all inside the phase $\psi_{2}$. For the Weyl point under consideration, $\left|c_{3}\right|>\left|d_{3}\right|$, and, hence, according to Eq. (C8), $\psi_{2}$ is a monotonically increasing function of $\beta$. Note that $\cos \chi=\left(\xi_{f} / r\right)$, with $\chi \in(0, \pi)$, so $\chi$ is a monotonically decreasing function of the working frequency. Then combined with Eqs. (C2) and (C6), we can conclude that $\psi_{2}$ is a monotonically increasing function of $\varphi$ while a monotonically decreasing function of the working frequency. Combined with the dependence of $\phi$ on $\psi_{2}$, we find $\phi$ is a monotonically decreasing function of the polar angle $\varphi$ and a monotonically increasing function of the working frequency. Combining Eqs. (C6) and (C8), we know that when $\varphi+\chi$ goes from $-\pi$ to $\pi, \psi_{2}$ varies from $-\pi / 2$ to $\pi / 2$. Meanwhile, Eq. (C14) tells us that when

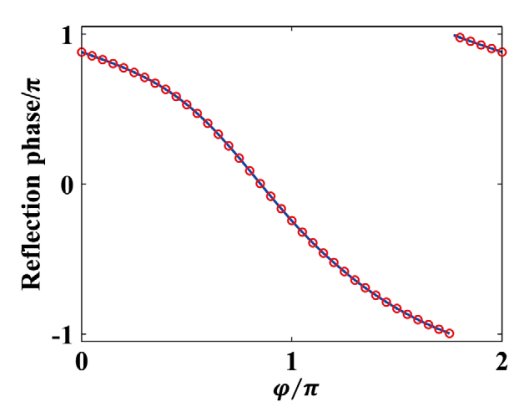

FIG. 8. The reflection phase as a function of the polar angle $\varphi$ on a circle with radius 0.01 in the $p-q$ space around the Weyl point. Blue solid line and red circles represent the reflection phases calculated through the transfer matrix method and Eq. (C14), respectively. Here, we consider the same Weyl point as in Fig. 6. The parameters of the PC are given by $d_{a}=97 \mathrm{~nm}$, $d_{b}=72 \mathrm{~nm}$, and the Weyl point considered is between the first and the second bands and located at $(p, q)=(0,0)$. The $\mathrm{PC}$ is truncated at the starting plane of the first layer, and the working frequency is also fixed at the frequency of the Weyl point, which is $f_{w}=247.6 \mathrm{THz}$. 
(a)

(b)
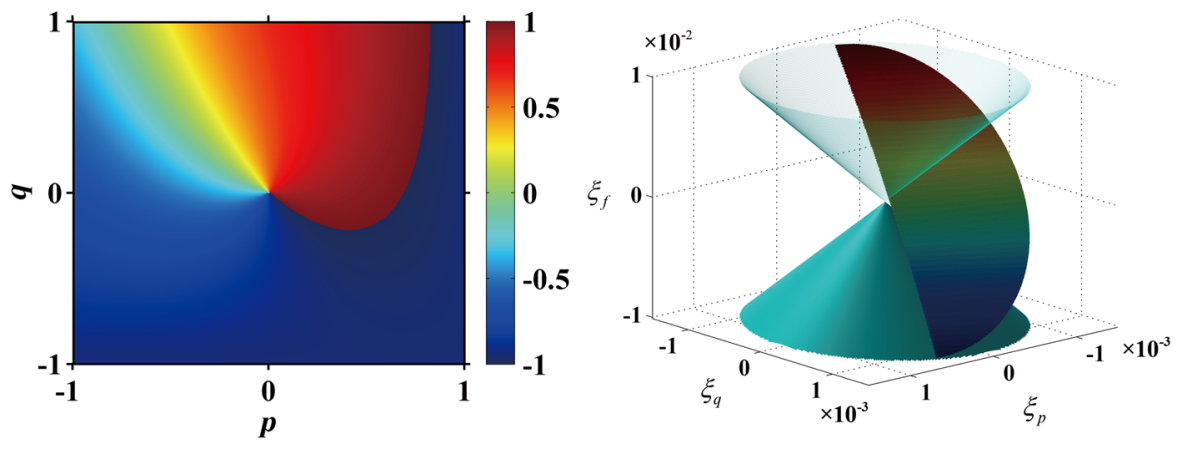

FIG. 9. (a) The reflection phase in the $p-q$ space for the PCs with $d_{a}=97 \mathrm{~nm}$ and $d_{b}=72 \mathrm{~nm}$. Now the PCs are truncated at the starting plane of the first layer. The working frequency is also fixed at the frequency of the Weyl point, which is $f_{w}=247.6 \mathrm{THz}$. Compared with Fig. 3(a), the reflection phase in the $p-q$ space changes locally, but the charge of the vortex located at the Weyl point stays the same. (b) The dispersion of the interface states near a Weyl point, where the cyan cone represents the bulk state and the colored sheet represents the interface states. Color code of the interface states represents different frequencies. In this calculation, the photonic crystals are truncated at the starting plane of the first layer.

$\psi_{1} / 2+\psi_{2}+k_{a} x-\pi / 4$ varies from $-\pi / 2$ to $\pi / 2$, the reflection phase goes from $\pi$ to $-\pi$. So we can conclude that when the polar angle $\varphi$ circles the Weyl point once, the reflection phase goes continuously from $\pi$ to $-\pi$ once. This explains the vortex structure of the reflection phase in the parameter space, and the charge of the vortex is the same as the charge of the Weyl point.

Also, changing the truncation position or the working frequency just shifts the reflection phases and does not change the conclusion above. As an example, we change the truncating position, and now the PCs are truncated at the starting plane of the first layer. The corresponding reflection phases in the $p-q$ space are shown in Fig. 9(a), where the parameters used are the same as those in Fig. 3(a), and the working frequency is also fixed at the frequency of the Weyl point. Compared with Fig. 3(a), the reflection phase in Fig. 9(a) changes locally, but the charge of the vortex is still preserved. If the truncating position of the PC is inside other layers, we can obtain the same conclusion following a similar proof as the one given above.

We now analyze Eq. (C14) in more detail. When $\xi_{f}=-r$, we have $\chi=\pi$, which is independent of the value of $r$, so the reflection phase maintains a constant for a fixed $\varphi$, while for $\xi_{f}=r$, we can also get the same result, but with $\chi=0$ instead. Now we consider the dispersion of the interface states between PCs and a mirror with reflection phase of 0 in the parameter space. As shown in Fig. 9(b), the interface states form a surface which rotates $180^{\circ}$ around the Weyl point as frequency increases. Such features were discussed before [56]; however, as effective Hamiltonians are used in Fig. 9(b), the intersection between the interface states and the bulk states is a straight line here.

In the above derivation, we use the transfer matrix method which is specialized to our systems. However, the existence of the reflection phase vortex pinning at the Weyl point is not specialized to our systems. To see this point, let us focus on the band gap region. In general, the surface property of a truncated bulk is characterized by the impedance matrix [57]. However, if the surface property is dominated by one plane wave component, then the surface property can be approximately described by a scalar surface impedance or equivalently the reflection phase [58]. Let us now assume that inside the boundary region between a system with Weyl points and a reflecting substrate there exist arclike surface states originating from one Weyl point. Note that these surface states should always exist regardless of the property of the reflecting substrate; hence, the reflection phase should be winding in the periodic $[-\pi \pi)$ when circling around the Weyl point. This indicates that the reflection phase vortex pining at the Weyl point is a general feature in a system possessing Weyl points.

[1] X. Wan, A. M. Turner, A. Vishwanath, and S. Y. Savrasov, Topological Semimetal and Fermi-Arc Surface States in the Electronic Structure of Pyrochlore Iridates, Phys. Rev. B 83, 205101 (2011).

[2] P. Hosur and X. Qi, Recent Developments in Transport Phenomena in Weyl Semimetals, C.R. Phys. 14, 857 (2013).

[3] B. Q. Lv et al., Experimental Discovery of Weyl Semimetal TaAs, Phys. Rev. X 5, 031013 (2015).

[4] S.-Y. Xu et al., Discovery of a Weyl Fermion Semimetal and Topological Fermi Arcs, Science 349, 613 (2015).

[5] A. A. Soluyanov, D. Gresch, Z. Wang, Q. Wu, M. Troyer, X. Dai, and B. A. Bernevig, Type-II Weyl Semimetals, Nature (London) 527, 495 (2015).

[6] K. Deng et al., Experimental Observation of Topological Fermi Arcs in Type-II Weyl Semimetal $\mathrm{MoTe}_{2}$, Nat. Phys. 12, 1105 (2016).

[7] T.-R. Chang et al., Prediction of an Arc-Tunable Weyl Fermion Metallic State in $\mathrm{Mo}_{x} \mathrm{~W}_{1-x} \mathrm{Te}_{2}$, Nat. Commun. 7, 10639 (2016). 
[8] S.-Y. Xu et al., Discovery of a Weyl Fermion State with Fermi Arcs in Niobium Arsenide, Nat. Phys. 11, 748 (2015).

[9] L. Lu, L. Fu, J. D. Joannopoulos, and M. Soljačić, Weyl Points and Line Nodes in Gyroid Photonic Crystals, Nat. Photonics 7, 294 (2013).

[10] J. Bravo-Abad, L. Lu, L. Fu, H. Buljan, and M. Soljačić, Weyl Points in Photonic-Crystal Superlattices, 2D Mater. 2, 034013 (2015).

[11] M. Xiao, Q. Lin, and S. Fan, Hyperbolic Weyl Point in Reciprocal Chiral Metamaterials, Phys. Rev. Lett. 117, 057401 (2016).

[12] W.-J. Chen, M. Xiao, and C. T. Chan, Photonic Crystals Possessing Multiple Weyl Points and the Experimental Observation of Robust Surface States, Nat. Commun. 7, 13038 (2016).

[13] M.-L. Chang, M. Xiao, W.-J. Chen, and C. T. Chan, Multiple Weyl Points and the Sign Change of Their Topological Charges in Woodpile Photonic Crystals, Phys. Rev. B 95, 125136 (2017).

[14] J. Noh, S. Huang, D. Leykam, Y. D. Chong, K. P. Chen, and M. C. Rechtsman, Experimental Observation of Optical Weyl Points and Fermi Arc-like Surface States, Nat. Phys. 13, 611 (2017).

[15] M. Xiao, W.-J. Chen, W.-Y. He, and C. T. Chan, Synthetic Gauge Flux and Weyl Points in Acoustic Systems, Nat. Phys. 11, 920 (2015).

[16] Z. Yang and B. Zhang, Acoustic Type-II Weyl Nodes from Stacking Dimerized Chains, Phys. Rev. Lett. 117, 224301 (2016).

[17] W. Gao, B. Yang, M. Lawrence, F. Fang, B. Beri, and S. Zhang, Photonic Weyl Degeneracies in Magnetized Plasma, Nat. Commun. 7, 12435 (2016).

[18] P. Roushan et al., Observation of Topological Transitions in Interacting Quantum Circuits, Nature (London) 515, 241 (2014).

[19] M. D. Schroer, M. H. Kolodrubetz, W. F. Kindel, M. Sandberg, J. Gao, M. R. Vissers, D. P. Pappas, A. Polkovnikov, and K. W. Lehnert, Measuring a Topological Transition in an Artificial Spin-1/2 System, Phys. Rev. Lett. 113, 050402 (2014).

[20] R.-P. Riwar, M. Houzet, J. S. Meyer, and Y. V. Nazarov, Multi-Terminal Josephson Junctions as Topological Matter, Nat. Commun. 7, 11167 (2016).

[21] O. Boada, A. Celi, J. I. Latorre, and M. Lewenstein, Quantum Simulation of an Extra Dimension, Phys. Rev. Lett. 108, 133001 (2012).

[22] L. Yuan, Y. Shi, and S. Fan, Photonic Gauge Potential in a System with a Synthetic Frequency Dimension, Opt. Lett. 41, 741 (2016).

[23] B. Lian and S.-C. Zhang, Five-Dimensional Generalization of the Topological Weyl Semimetal, Phys. Rev. B 94, 041105 (2016).

[24] F. Mei, Z.-Y. Xue, D.-W. Zhang, L. Tian, C. Lee, and S.-L. Zhu, Witnessing Topological Weyl Semimetal Phase in a Minimal Circuit-QED Lattice, Quantum Sci. Technol. 1, 015006 (2016).

[25] Q. Lin, M. Xiao, L. Yuan, and S. Fan, Photonic Weyl Point in a Two-Dimensional Resonator Lattice with a Synthetic Frequency Dimension, Nat. Commun. 7, 13731 (2016).
[26] L. Lu, Z. Wang, D. Ye, L. Ran, L. Fu, J. D. Joannopoulos, and M. Soljačić, Experimental Observation of Weyl Points, Science 349, 622 (2015).

[27] L. Lu and Z. Wang, Topological One-Way Fiber of Second Chern Number, arXiv:1611.01998.

[28] Z. Fang et al., The Anomalous Hall Effect and Magnetic Monopoles in Momentum Space, Science 302, 92 (2003).

[29] N. Yu, P. Genevet, M. A. Kats, F. Aieta, J.-P. Tetienne, F. Capasso, and Z. Gaburro, Light Propagation with Phase Discontinuities: Generalized Laws of Reflection and Refraction, Science 334, 333 (2011).

[30] M. Xiao, Z. Q. Zhang, and C. T. Chan, Surface Impedance and Bulk Band Geometric Phases in One-Dimensional Systems, Phys. Rev. X 4, 021017 (2014).

[31] Q. Wang, M. Xiao, H. Liu, S. Zhu, and C. T. Chan, Measurement of the Zak Phase of Photonic Bands through the Interface States of a Metasurface/Photonic Crystal, Phys. Rev. B 93, 041415 (2016).

[32] M. S. Rudner, N. H. Lindner, E. Berg, and M. Levin, Anomalous Edge States and the Bulk-Edge Correspondence for Periodically Driven Two-Dimensional Systems, Phys. Rev. X 3, 031005 (2013).

[33] G. Lheureux, S. Azzini, C. Symonds, P. Senellart, A. Lemaitre, C. Sauvan, J. P. Hugonin, J. J. Greffet, and J. Bellessa, Polarization-Controlled Confined Tamm Plasmon Lasers, ACS Photonics 2, 842 (2015).

[34] C. Symonds, G. Lheureux, J. P. Hugonin, J. J. Greffet, J. Laverdant, G. Brucoli, A. Lemaitre, P. Senellart, and J. Bellessa, Confined Tamm Plasmon Lasers, Nano Lett. 13, 3179 (2013).

[35] C. Symonds, A. Lemaitre, P. Senellart, M. H. Jomaa, S. A. Guebrou, E. Homeyer, G. Brucoli, and J. Bellessa, Lasing in a Hybrid GaAs/Silver Tamm Structure, Appl. Phys. Lett. 100, 121122 (2012).

[36] O. Gazzano, S. M. de Vasconcellos, K. Gauthron, C. Symonds, P. Voisin, J. Bellessa, A. Lemaitre, and P. Senellart, Single Photon Source Using Confined Tamm Plasmon Modes, Appl. Phys. Lett. 100, 232111 (2012).

[37] O. Gazzano, S. M. de Vasconcellos, K. Gauthron, C. Symonds, J. Bloch, P. Voisin, J. Bellessa, A. Lemaître, and P. Senellart, Evidence for Confined Tamm Plasmon Modes Under Metallic Microdisks and Application to the Control of Spontaneous Optical Emission, Phys. Rev. Lett. 107, 247402 (2011).

[38] Z.-Y. Yang, S. Ishii, T. Yokoyama, T. D. Dao, M.-G. Sun, T. Nagao, and K.-P. Chen, Tamm Plasmon Selective Thermal Emitters, Opt. Lett. 41, 4453 (2016).

[39] H. Lu, X. T. Gan, B. H. Jia, D. Mao, and J. L. Zhao, Tunable High-Efficiency Light Absorption of Monolayer Graphene via Tamm Plasmon Polaritons, Opt. Lett. 41, 4743 (2016).

[40] V. Villafane, A. E. Bruchhausen, B. Jusserand, P. Senellart, A. Lemaitre, and A. Fainstein, Confinement of Gigahertz. Sound and Light in Tamm Plasmon Resonators, Phys. Rev. B 92, 165308 (2015).

[41] B. Auguie, M. C. Fuertes, P. C. Angelome, N. L. Abdala, G. Illia, and A. Fainstein, Tamm Plasmon Resonance in Mesoporous Multilayers: Toward a Sensing Application, ACS Photonics 1, 775 (2014).

[42] Y. K. Chen, D. Zhang, D. Qiu, L. Zhu, S. Yu, P. Yao, P. Wang, H. Ming, R. Badugu, and J. R. Lakowicz, 
Back Focal Plane Imaging of Tamm Plasmons and Their Coupled Emission, Laser Photonics Rev. 8, 933 (2014).

[43] G. Bräunlich, G. M. Graf, and G. Ortelli, Equivalence of Topological and Scattering Approaches to Quantum Pumping, Commun. Math. Phys. 295, 243 (2010).

[44] I. C. Fulga, F. Hassler, and A. R. Akhmerov, Scattering Theory of Topological Insulators and Superconductors, Phys. Rev. B 85, 165409 (2012).

[45] H. Wang, L. Zhou, and Y. D. Chong, Floquet Weyl Phases in a Three-Dimensional Network Model, Phys. Rev. B 93, 144114 (2016).

[46] Z. Yan and Z. Wang, Tunable Weyl Points in Periodically Driven Nodal Line Semimetals, Phys. Rev. Lett. 117, 087402 (2016).

[47] H. B. Nielsen and M. Ninomiya, Absence of Neutrinos on a Lattice, Nucl. Phys. B193, 173 (1981).

[48] N. D. Mermin, The Topological Theory of Defects in Ordered Media, Rev. Mod. Phys. 51, 591 (1979).

[49] See Supplemental Material at http://link.aps.org/ supplemental/10.1103/PhysRevX.7.031032 which includes the detailed experimental setup for measuring the reflection phase, the experimental setup for measuring the interface states and a discussion on the robustness of the "Fermi arc like interface states". A discussion on the absorption of interface states is also included.

[50] C. Fang, Y. Chen, H.-Y. Kee, and L. Fu, Topological Nodal Line Semimetals with and without Spin-Orbital Coupling, Phys. Rev. B 92, 081201 (2015).
[51] K. F. MacDonald, Z. L. Samson, M. I. Stockman, and N. I. Zheludev, Ultrafast Active Plasmonics, Nat. Photonics 3, 55 (2009).

[52] D. A. Fuhrmann, S. M. Thon, H. Kim, D. Bouwmeester, P. M. Petroff, A. Wixforth, and H. J. Krenner, Dynamic Modulation of Photonic Crystal Nanocavities Using Gigahertz Acoustic Phonons, Nat. Photonics 5, 605 (2011).

[53] S. Forstner, S. Prams, J. Knittel, E. D. van Ooijen, J. D. Swaim, G. I. Harris, A. Szorkovszky, W. P. Bowen, and H. Rubinsztein-Dunlop, Cavity Optomechanical Magnetometer, Phys. Rev. Lett. 108, 120801 (2012).

[54] C. Sheng, H. Liu, S. Zhu, and D. A. Genov, Active Control of Electromagnetic Radiation through an Enhanced Thermo-Optic Effect, Sci. Rep. 5, 8835 (2015).

[55] C. Fang, M. J. Gilbert, X. Dai, and B. A. Bernevig, MultiWeyl Topological Semimetals Stabilized by Point Group Symmetry, Phys. Rev. Lett. 108, 266802 (2012).

[56] C. Fang, L. Lu, J. Liu, and L. Fu, Topological Semimetals with Helicoid Surface States, Nat. Phys. 12, 936 (2016).

[57] F. J. Lawrence, L. C. Botten, K. B. Dossou, R. C. McPhedran, and C.M. de Sterke, Photonic-Crystal Surface Modes Found from Impedances, Phys. Rev. A 82, 053840 (2010).

[58] X. Huang, M. Xiao, Z.-Q. Zhang, and C. T. Chan, Sufficient Condition for the Existence of Interface States in Some TwoDimensional Photonic Crystals, Phys. Rev. B 90, 075423 (2014). 